\title{
Increased Number of White Matter Lesions in Patients with Familial Cerebral Cavernous Malformations
}

\author{
M.J. Golden, L.A. Morrison, H. Kim, and B.L. Hart
}

\begin{abstract}
BACKGROUND AND PURPOSE: Familial cerebral cavernous malformations, an autosomal dominant disorder, result in excess morbidity and mortality in affected patients. The disorder is most prevalent in the Southwest United States, where the affected families are most often carriers of the CCMT-KRITI Common Hispanic Mutation. The brain and spinal cord parenchyma in these individuals is usually affected by multiple cavernous malformations. Previous studies have shown abnormalities of endothelial cell junctions and the blood-brain barrier in cerebral cavernous malformations. Endothelial cell abnormalities have also been described in pathologic studies of white matter hyperintensities. We compared the prevalence of white matter hyperintensities in a population with known familial cerebral cavernous malformations.
\end{abstract}

MATERIALS AND METHODS: We examined 191 subjects with familial cerebral cavernous malformations who were enrolled into an institutional review board-approved study. All carry the same Common Hispanic Mutation in the CCM1 gene. Each subject underwent 3T MR imaging, including gradient recalled-echo, SWI, and FLAIR sequences. The number of cavernous malformations and the number of nonhemorrhagic white matter hyperintensities were counted. Subjects older than 60 years of age were excluded due to the high prevalence of white matter lesions in this population, and children younger than 6 were excluded due to potential sedation requirements. Logistic regression analysis was performed to determine the prevalence of abnormal white matter hyperintensities in those with familial cerebral cavernous malformations compared with healthy controls or those with sporadic cerebral cavernous malformation within the familial cerebral cavernous malformations group; it was also performed to evaluate the associations between abnormal white matter hyperintensities and age, sex, headaches, thyroid disease, diabetes, hypertension, hyperlipidemia, seizure history, or modified Rankin Scale score.

RESULTS: Familial CCM1 carriers have a higher prevalence of abnormal white matter hyperintensities (15.4\%) compared with both control populations ( $2.1 \%$ and $2.5 \%$, respectively) $(P<.05)$. Logistic regression showed no statistical association with sex, headaches, hyperlipidemia, hypertension, thyroid disease, seizure history, number of cerebral cavernous malformations, or modified Rankin Scale score among those with familial cerebral cavernous malformation. An expected correlation with age was shown.

CONCLUSIONS: Familial CCM7 carriers have not only an increased number of cerebral cavernous malformations but also an increased number of white matter T2 hyperintensities, spatially distinct from cerebral cavernous malformations, which exceeded that of a healthy population. Clinical findings did not explain the association with abnormal white matter hyperintensities in the familial cerebral cavernous malformation population. To our knowledge, these relationships have not been previously reported. This finding suggests an additional manifestation of endothelial abnormalities in this population.

ABBREVIATIONS: $C C M=$ cerebral cavernous malformation; $\mathrm{fCCM}=$ familial cerebral cavernous malformations; $\mathrm{WMHI}=$ white matter hyperintensities

Eamilial cerebral cavernous malformations (fCCM) constitute an autosomal dominant disorder with variable penetrance. ${ }^{1}$
From the Departments of Radiology (M.J.G., B.L.H.) and Neurology (L.A.M.), University of New Mexico, Albuquerque, New Mexico; and Departments of Anesthesiology and Perioperative Care (H.K.) and Epidemiology and Biostatistics (H.K.), University of California, San Francisco, San Francisco, California.

Dr Golden was supported as a trainee by the Brain Vascular Malformation Consortium. The Brain Vascular Malformation Consortium is supported by a grant from the National Institutes of Health (U54 NS065705) and is a part of the National Institutes of Health Rare Disease Clinical Research Network, supported through a collaboration between the National Institutes of Health Office of Rare Diseases
This condition occurs throughout the world but is especially prevalent in the Southwestern United States. ${ }^{2,3}$ Repeated hemorrhages cause significant morbidity and mortality in affected indi-

Research at the National Center for Advancing Translational Science and the $\mathrm{Na}$ tional Institute of Neurologic Disorders and Stroke.

Paper previously presented at: Annual Meeting of the American Society of Neuroradiology and the Foundation of the ASNR Symposium, May 17-22, 2014; Montreal, Quebec, Canada. Please address correspondence to Blaine L. Hart, MD, Department of Radiology, MSC10 5530, 1 University of New Mexico, Albuquerque, NM 87131-0001; e-mail: bhart@salud.unm.edu

- Indicates open access to non-subscribers at www.ajnr.org

http://dx.doi.org/10.3174/ajnr.A4200 


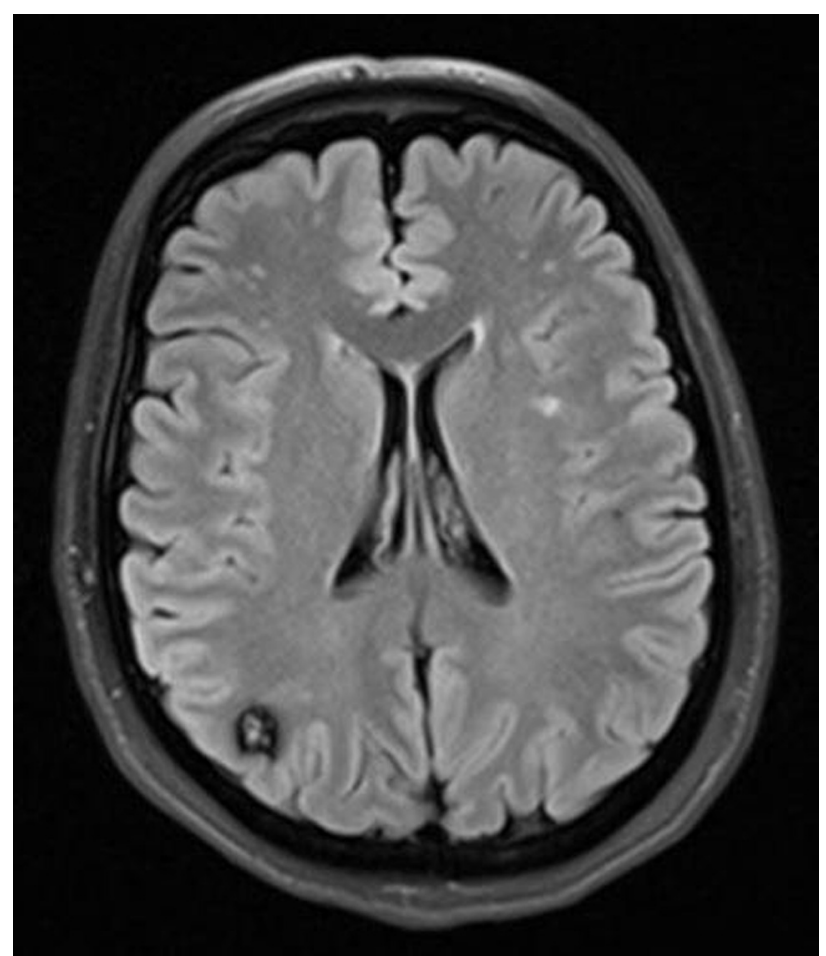

FIG 1. FLAIR images obtained in a 39-year-old patient with fCCM demonstrate a large number of WMHI. Also note the CCM in the right parietal lobe.

viduals and families. Patients present with hemorrhage, seizures, headaches, and/or focal neurologic deficits, and some patients ultimately die from associated complications.

Imaging evaluation of these patients often focuses on the presence, location, and size of the vascular malformations. ${ }^{2,4}$ Bloodbreakdown products within the cerebral cavernous malformations (CCMs) are best evaluated with susceptibility-sensitive MR imaging, specifically SWI and T2 gradient recalled-echo sequences. However, FLAIR and T2-weighted sequences sometimes disclose additional findings. We have noted an unexpected number of T2 white matter hyperintensities (WMHI) in some of these patients (Figs 1 and 2). Although such WMHI are generally considered nonspecific on MR imaging, the association of WMHI with a wide variety of conditions with small-vessel disease raised the question of their significance in familial CCM. We tested the hypothesis that increased WM lesions are present in patients with fCCM. We compared the prevalence of T2 WHMI in patients with fCCM with those in a healthy control group and with patients with sporadic CCMs and evaluated whether confounding factors related to small-vessel disease could explain this association.

\section{MATERIALS AND METHODS}

All parts of this study were performed with institutional review board approval. After informed consent, 191 subjects were enrolled into an ongoing study of fCCM with the Common Hispanic Mutation (Q455X mutation) recruited as part of the Brain Vascular Malformation Consortium CCM Project. ${ }^{5}$ Brain MR imaging at 3T was performed on all subjects, including T2 gradient recalled-echo, T2 FSE (both with 3-mm section thickness),

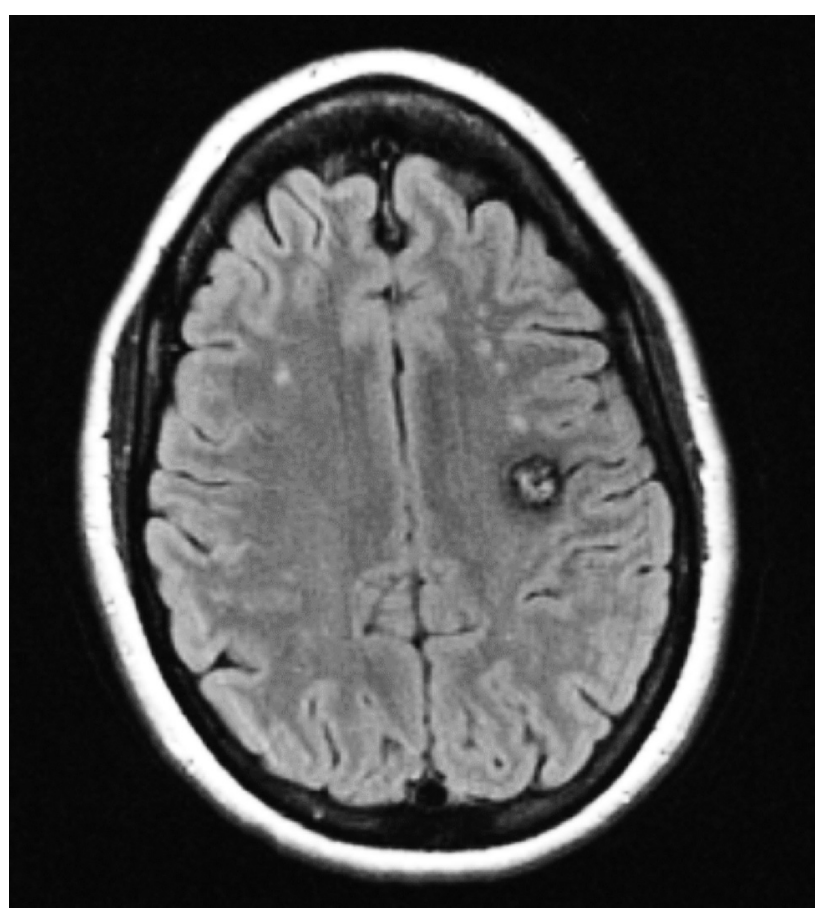

FIG 2. FLAIR images from this 26-year-old patient show numerous WMHI in addition to the CCM located in the posterior left frontal lobe.

SWI (1.5-mm section thickness), and FLAIR imaging (4-mm section thickness). CCMs were counted and characterized on the SWI and gradient recalled-echo sequences by a neuroradiologist with extensive CCM research experience as part of the overall study plan. Children younger than of 6 years of age were not eligible to participate in this study due to the potential need for sedation for MR imaging.

WMHI were counted on the FLAIR sequences, with concurrent reference to T2 sequences. All subjects older than 60 years of age (28 total) were excluded from this retrospective review of white matter lesions, given the known high correlation of WMHI with advancing age. ${ }^{6,7}$ One subject was excluded because the study MR imaging was terminated before FLAIR imaging; 149 subjects remained ( 56 male, 93 female; $6-59$ years of age). There is no universally accepted measure of a "normal" number of white matter changes. They are known to increase with age; indeed, age is the strongest factor associated with WMHI. A common rule of thumb is that $1 \mathrm{WMHI}$ per decade of life is considered unremarkable. $^{8}$ Guidelines for normal or unremarkable WMHI become less useful with increasing patient age. For purposes of this study, the number of WMHI was considered abnormally high if there were $>5$, or unusually large or confluent lesions were seen in any individual younger than 40 years of age, or $>10$ lesions were seen in anyone between 40 and 60 years of age.

A control group of 47 healthy volunteers younger than 60 years of age who gave informed consent and were carefully screened to eliminate a history of disease were evaluated for WMHI. This control population was evaluated on the same 3T magnet, and demographics closely resembled those of the fCCM population.

Additionally, a group of 39 patients younger than 60 years of 
Table 1: Frequency distribution of WHMI in patients with $\mathrm{fCCM}$

\begin{tabular}{lcr}
\hline WMHI No. & Frequency & $\%$ \\
\hline 0 & 88 & $59 \%$ \\
1 & 14 & $9 \%$ \\
2 & 10 & $7 \%$ \\
$3-10$ & 20 & $13 \%$ \\
$11-20$ & 3 & $2 \%$ \\
$21-30$ & 5 & $3 \%$ \\
$31-40$ & 5 & $3 \%$ \\
$41-50$ & 2 & $1 \%$ \\
$51+$ & 2 & $1 \%$ \\
\hline
\end{tabular}

age (including both 3T and 1.5T MR imaging, part of an institutional review board-approved study) was identified as having sporadic CCMs; each patient had only 1 lesion present in the brain and lacked clinical or family history evidence of fCCM. Any patient with multiple CCMs was excluded from this control group because this finding was suspicious for a familial pattern of CCMs (Common Hispanic Mutation or otherwise). FLAIR imaging was then evaluated, and abnormal white matter lesions were counted.

For the control groups, all FLAIR images were reviewed by a neuroradiology fellow and the same neuroradiologist who originally read the fCCM MR imaging studies. Infrequent differences were resolved by a consensus read. The prevalence of abnormal WMHI in patients with $\mathrm{fCCM}$ was compared with that in the 2 control groups by using logistic regression to account for age and sex.

Descriptive statistics, including proportions for categoric variables and means, SDs, ranges, and interquartile ranges for continuous variables, were computed. Logistic regression analysis was preformed to evaluate whether the abnormal status of WMHI differed between patients with fCCM and the 2 control groups while adjusting for age and sex; results are reported as odds ratios with associated 95\% confidence intervals. Among patients with fCCM only, the Fisher exact test (categoric variable) or Wilcoxon rank sum test (continuous variable) was performed to compare the univariate association of abnormal WMHI with the number of CCMs, age, sex, hyperlipidemia (physician diagnosed or taking medication), hypertension (physician diagnosed or the subject taking medications at the initial examination ${ }^{9}$ ), headaches, seizures, thyroid disease, diabetes, and modified Rankin Scale score as a measure of overall neurologic disability. The distribution of CCM and WMHI lesions is heavily right-skewed; thus, values were log-transformed before parametric analysis to avoid the effects of overly influential observations. Clinical factors found to have a $P \leq .10$ in univariate analysis were tested in a multivariate logistic regression model adjusting for age and sex. All $P$ values reported are 2 -sided, and we considered $P<.05$ to be statistically significant.

\section{RESULTS}

Twenty-three of 149 (15.4\%) patients in the fCCM group (mean age, 33.1 years; range, 6-59 years) had an abnormal number of WMHI. Healthy control subjects (mean age, 31.3 years; range, 21-46) had a prevalence of $2.1 \%$ (1/48) (Table 1). Patients with sporadic CCM (mean age, 28.1 years; range, 4-56 years) had white matter abnormalities at a prevalence of $2.5 \%(1 / 39)$. Thus,
Table 2: Association of abnormal WMHI and characteristics in 149 patients with $\mathrm{fCCM}^{\mathrm{a}}$

\begin{tabular}{|c|c|c|c|}
\hline fCCM Characteristic & $\begin{array}{c}\text { Abnormal } \\
\text { WMHI } \\
(n=23)\end{array}$ & $\begin{array}{c}\text { Normal } \\
\text { WMHI } \\
(n=126)\end{array}$ & $P$ Value \\
\hline \multicolumn{4}{|l|}{$\operatorname{Sex}($ No.) $(\%)$} \\
\hline Female & $18(78)$ & $75(60)$ & .11 \\
\hline Male & $5(22)$ & $51(40)$ & \\
\hline Age (yr) (mean) (SD) & $23(41)$ & $32(16)$ & .01 \\
\hline \multicolumn{4}{|l|}{ Headaches (No.) (\%) } \\
\hline Yes & $15(65)$ & $79(64)$ & 1.00 \\
\hline No & $8(35)$ & $45(36)$ & \\
\hline \multicolumn{4}{|l|}{ Seizures (No.) (\%) } \\
\hline Yes & $11(48)$ & $48(39)$ & .49 \\
\hline No & $12(52)$ & $76(61)$ & \\
\hline \multicolumn{4}{|l|}{ Thyroid (No.) (\%) } \\
\hline Yes & $3(13)$ & $9(7)$ & .40 \\
\hline No & $20(87)$ & $115(93)$ & \\
\hline \multicolumn{4}{|l|}{ Diabetes (No.) (\%) } \\
\hline Yes & $2(9)$ & $12(10)$ & 1.00 \\
\hline No & $21(91)$ & $112(90)$ & \\
\hline \multicolumn{4}{|l|}{ Hyperlipidemia (No.) (\%) } \\
\hline Yes & $7(30)$ & $17(13)$ & .06 \\
\hline No & $16(70)$ & $109(87)$ & \\
\hline \multicolumn{4}{|l|}{ Hypertension (No.) (\%) } \\
\hline Yes & $6(26)$ & $15(12)$ & .10 \\
\hline No & $17(74)$ & $109(88)$ & \\
\hline \multicolumn{4}{|c|}{ Modified Rankin Scale (No.) (\%) } \\
\hline 0 & $4(17)$ & $46(37)$ & .33 \\
\hline 1 & $10(44)$ & $37(30)$ & \\
\hline 2 & $7(30)$ & $27(22)$ & \\
\hline 3 & $2(9)$ & $11(9)$ & \\
\hline 4 & $0(0)$ & $2(2)$ & \\
\hline \multicolumn{4}{|l|}{ CCM lesions } \\
\hline Median (IQR) & $29(29)$ & $11(39)$ & .08 \\
\hline Mean (SD) & $60(122)$ & $42(96)$ & \\
\hline
\end{tabular}

Note:- IQR indicates interquartile range.

a Note that some numbers for characteristics do not add up to column totals due to missing data.

the prevalence of abnormal WMHI was significantly higher in the fCCM group compared with either control group (1-sided $P<$ $.05)$.

The prevalence of abnormal WMHI in the healthy control group was nearly identical to the sporadic CCM rate. These 2 populations were then pooled together and compared with the fCCM population. The age and sex distribution were similar between the groups, but logistic regression was further used to evaluate the rate of abnormal WMHI between the fCCM and the pooled control group to correct for sex and age. The odds ratio of patients with fCCM having abnormal quantities of WMHI was 8.3 (95\% CI, 1.03-66.9) compared with the combined group, and it was statistically significant $(P<.05)$.

Second, we evaluated potential clinical factors within the fCCM group that could explain the increased prevalence of abnormal WMHI (Table 2). No statistically significant association was found with sex $(P=.11)$, headaches $(P=1.00)$, seizure $(P=.49)$, thyroid disease $(P=.40)$, hyperlipidemia $(P=.06)$, diabetes $(P=1.00)$, or hypertension $(P=.10)$. Age did demonstrate a positive association $(P=.01)$, an expected result. We found no association of abnormal WMHI with neurologic disability as measured by the modified Rankin Scale score $(P=.33)$. 
The patients with fCCM with abnormal WMHI tended to have a greater number of CCM lesions, though this was not statistically significant $(P=.08)$. Including age in a logistic regression model explains away the association between WMHI and the number of CCMs $(P=.98)$, likely because both WMHI and CCM lesions are strongly correlated with increasing age. A multivariable logistic regression model with hyperlipidemia, hypertension, number of CCMs, age, and sex as predictors of abnormal WMHI status yielded no statistically significant associations.

\section{DISCUSSION}

Our study showed a significant increase in WMHI in patients with fCCM compared with healthy controls and with patients with sporadic CCM, which, to the best of our knowledge, has not been previously reported. Bright spots on T2-weighted MR images have been an uncertainty in brain imaging since the inception of MR imaging. This challenge is reflected in the various terms that have been used such as white matter hyperintensities (a purely descriptive term), leukoaraiosis, chronic microvascular changes, chronic microangiopathy, white matter changes of aging, and unidentified bright objects. Such lesions are known to correlate with patient age and can additionally be caused by demyelination, edema, and gliosis, including causes of microvascular change such as hypertension, lupus erythematosus, migraines, seizures, or other vasculopathies. FLAIR hyperintensities may sometimes accompany perivascular spaces. Although some of the WMHI in this study could be associated with perivascular spaces, typical radially oriented perivascular spaces were generally distinguishable by using both FSE T2 and FLAIR on the 3T system. Moreover, changes associated with perivascular spaces are likely to affect all groups in the study to a similar degree. Considerable research has been done on the significance of WMHI in the elderly population. Volumetric measurements have often been used in research studies of elderly patients, in whom the T2 changes are frequently confluent. The presence of focal WMHI in a young or middle-aged population is a common finding in the routine clinical practice of radiology.

Interpretation of the literature on this finding is challenging because of the varying technical factors used in different reports, including field strength and MR imaging sequences used, as well as varying subject ages; such factors are not always specified in the literature. The frequency of WMHI varies widely in the literature, but several authors reported a rate between $0.5 \%$ and $5 \%$ for a young healthy population. ${ }^{10,11}$ We were able to use a control group of healthy volunteers who were scanned on the same MR imaging system as our fCCM study group, avoiding some of the technical limitations of historical controls. The findings of the additional control group from clinical studies of patients with sporadic CCM appeared very similar to those of the healthy control group, despite a mix of 3T and 1.5T studies. Both groups thus appear very similar to those in literature reports of healthy subjects, but our study confers the added advantage of clearly defined and comparable technical factors.

A variety of pathologic correlates likely exist for the T2 hyperintensities seen on MR imaging. However, we examined possible causes of microvascular changes for association with abnormal WMHI within the fCCM group, and none were found to be sig- nificantly associated with abnormal WMHI status. Seizure disorders are reported to be associated with increased numbers of WMHI, ${ }^{12-14}$ and seizures are common complications of CCMs. However, the prevalence of WMHI within the fCCM group was nearly identical for those with and without seizure history.

Hypertension and hyperlipidemia were both present at higher frequencies in patients with fCCM with abnormal WMHI but were not found to be significant predictors of abnormal WMHI status in either univariate or multivariate analysis. Both of these variables were derived from patient self-reports of either physician diagnosis or the patient taking medications for these conditions. We did not have detailed information available on the length of the diagnosis or the details of treatment, which may have limited our ability to detect significant associations with these clinically important variables.

Although we could not find an explanation for the increased prevalence of abnormal WMHI with typical clinical factors of microvascular changes, it is well-known that patients with fCCM have underlying endothelial dysfunction. ${ }^{15-17}$ Additionally, CCMs demonstrate abnormal ultrastructural characteristics, ${ }^{18}$ and this abnormal physiology is known to result in a compromised blood-brain barrier. ${ }^{19}$ In vivo human permeability studies have shown that the transfer rate (influx constant) is increased in CCMs. ${ }^{20}$ Reviews of neuropathologic information about WMHI in older individuals (not those with fCCM) have shown structural abnormalities and compromise of the blood-brain barrier. ${ }^{21}$ The influx constant has also been shown to be increased in nonspecific WMHI in brain white matter in permeability studies of subjects with vascular cognitive impairment. ${ }^{20}$ Endothelial abnormalities caused by the mutations in fCCM may be a factor in WMHI seen in patients with fCCM.

The increased prevalence of WMHI in FCCM is modest and is not clearly correlated with the number of CCM lesions, especially after accounting for age. Moreover, we did not find a correlation of WMHI with disability as measured by the modified Rankin Scale score. Our definition of abnormal WMHI served as a pragmatic and conservative measure for this study. Although we have not observed T2 WMHI to precede the formation of CCMs, a causal relationship cannot be excluded, and further longitudinal studies will be needed. It is possible that endothelial abnormalities in fCCM predispose to a higher risk of WMHI when combined with other, more common risk factors for microvascular disease. Clinically, when unexpected numbers of WMHI are observed in patients with fCCM, common causes such as hypertension should be considered. If such factors are not found, however, it appears that the fCCM condition itself may be associated with increased WMHI.

The results of our study, combined with historical studies, lead us to hypothesize that underlying endothelial abnormalities in the fCCM population could result in increased permeability with resultant increased hemorrhage, new lesion formation of cerebral cavernous malformations, and increased formation of WMHI. These 2 separate findings may be different manifestations of a common underlying process. Future neuropathologic studies and/or in vivo permeability studies will offer further insight into the mechanisms for these findings. 


\section{CONCLUSIONS}

Subjects with fCCM have an increased prevalence of abnormal WMHI compared with healthy control populations and those with sporadic CCMs. Additionally, no single clinical factor could be associated with patients with fCCM. We found no association between abnormal WMHI and sex, hypertension, headache, thyroid disease, seizures, diabetes or hyperlipidemia, number of CCMs, or modified Rankin Scale scores among patients with fCCM. CCMs and WMHI may be separate and distinct outcomes of underlying endothelial dysfunction in patients with fCCM.

\section{ACKNOWLEDGMENTS}

We thank Herbert Davis and Ron Schrader from the University of New Mexico and Jeffrey Nelson from the University of California, San Francisco, for help with statistical analysis; Cheryl Aine, MD, of the University of New Mexico for providing data from healthy control volunteers; and Beth Baca, MSW, for patient recruitment, coordination, and data management.

Disclosures: Michael J. Golden—RELATED: Grant: National Institutes of Health,* Comments: This research was funded through a National Institutes of Health grant; Employment: University of New Mexico, Comments: salary as a PGY-7 Neuroradiology Fellow. Leslie A. Morrison—RELATED: Grant: National Institute of Neurologic Disorders and Stroke/National Institutes of Health.* Blaine L. Hart-RELATED: Grant: National Institutes of Health (U54 NS065705).* Helen Kim—RELATED: Grant: National Institutes of Health, Comments: support received individually and by institution. * Money paid to the institution.

\section{REFERENCES}

1. Rivera PP, Willinsky RA, Porter PJ. Intracranial cavernous malformations. Neuroimaging Clin N Am 2003;13:27-40

2. Kattapong VJ, Hart BL, Davis LE. Familial cerebral cavernous angiomas: clinical and radiologic studies. Neurology 1995;45:492-97

3. Gunel M, Awad IA, Finberg K, et al. A founder mutation as a cause of cerebral cavernous malformation in Hispanic Americans. $N$ Engl JMed 1996;334:946-51

4. Zabramski JM, Wascher TM, Spetzler RF, et al. The natural history of familial cavernous malformations: results of an ongoing study. J Neurosurg 1994;80:422-32

5. Akers AL, Ball KL, Clancy M, et al. Brain vascular malformation consortium: overview, progress and future directions. J Rare Disord 2013;1:5

6. Christiansen P, Larsson HB, Thomsen C, et al. Age dependent white matter lesions and brain volume changes in healthy volunteers. Acta Radiol 1994;35:117-22

7. Vernooij MW, Ikram MA, Tanghe HL, et al. Incidental findings on brain MRI in the general population. $N$ Engl J Med 2007;357: 1821-28

8. Osborn AG. Osborn's Brain: Imaging, Pathology, and Anatomy. Salt Lake City: Amirsys; 2012

9. Liao D, Cooper L, Cai J, et al. Presence and severity of cerebral white matter lesions and hypertension, its treatment, and its control: the ARIC Study-Atherosclerosis Risk in Communities Study. Stroke 1996;27:2262-70

10. Hopkins RO, Beck CJ, Burnett DL, et al. Prevalence of white matter hyperintensities in a young healthy population. J Neuroimaging 2006;16:243-51

11. Katzman GL, Dagher AP, Patronas NJ. Incidental findings on brain magnetic resonance imaging from 1000 asymptomatic volunteers. JAMA 1999;282:36-39

12. Eriksson SH, Stepney A, Symms MR, et al. Ultra-fast low-angle rapid acquisition and relaxation enhancement (UFLARE) in patients with epilepsy. Neuroradiology 2001;43:1040-45

13. Jansen JFA, Vlooswijk MC, Majoie HM, et al. White matter lesions in patients with localization-related epilepsy. Invest Radiol 2008;43: $552-58$

14. Wieshmann UC. Clinical application of neuroimaging in epilepsy. J Neurol Neurosurg Psychiatry 2003;74:466-70

15. Stockton RA, Shenkar R, Awad IA, et al. Cerebral cavernous malformations proteins inhibit Rho kinase to stabilize vascular integrity. $J$ Exp Med 2010;207:881-96

16. Glading A, Han J, Stockton RA, et al. KRIT-1/CCM1 is a Rap1 effector that regulates endothelial cell cell junctions. J Cell Biol 2007; 179:247-54

17. Schneider H, Errede M, Ulrich NH, et al. Impairment of tight junctions and glucose transport in endothelial cells of human cerebral cavernous malformations. J Neuropathol Exp Neurol 2011;70:417-29

18. Tu J, Stoodley MA, Morgan MK, et al. Ultrastructural characteristics of hemorrhagic, nonhemorrhagic, and recurrent cavernous malformations. J Neurosurg 2005;103:903-09

19. Clatterbuck RE, Eberhart CG, Crain BJ, et al. Ultrastructural and immunocytochemical evidence that an incompetent blood-brain barrier is related to the pathophysiology of cavernous malformations. J Neurol Neurosurg Psychiatry 2001;71:188-92

20. Hart BL, Taheri S, Rosenberg GA, et al. Dynamic contrast-enhanced MRI evaluation of cerebral cavernous malformations. Trans Stroke Res 2013;4:500-06

21. Young VG, Halliday GM, Kril JJ. Neuropathologic correlates of white matter hyperintensities. Neurology 2008;71:804-11 